\title{
Structural Basis for Clinical Longevity of Carbapenem Antibiotics in the Face of Challenge by the Common Class A $\beta$-Lactamases from the Antibiotic-Resistant Bacteria
}

\author{
Laurent Maveyraud, ${ }^{\dagger}$ Lionel Mourey,${ }^{\dagger}$ Lakshmi P. Kotra, ${ }^{\ddagger}$ Jean-Denis Pedelacq,${ }^{\dagger}$ \\ Valérie Guillet, ${ }^{\dagger}$ Shahriar Mobashery, ${ }^{*, *}$ and Jean-Pierre Samama*,†
}

\begin{abstract}
Groupe de Cristallographie Biologique, Institut de Pharmacologie et de Biologie Structurale du CNRS, 205 route de Narbonne, 31077-Toulouse Cedex, France, and Department of Chemistry, Wayne State University, Detroit, Michigan 48202-3489
\end{abstract}

Received May 26, 1998

\begin{abstract}
Bacteria resistant to antibiotics are being selected in a relatively short time, and cases of infections resistant to treatment by all known antibiotics are being identified at alarming rates. The primary mechanism for resistance to $\beta$-lactam antibiotics is the catalytic function of $\beta$-lactamases. However, imipenem (a $\beta$-lactam) resists the action of most $\beta$-lactamases and is virtually the last effective agent against the vancomycin-resistant Gram-positive bacteria, as well as against multiple antibiotic-resistant Gram-negative organisms. Here, we report the crystal structure, to $1.8 \AA$ resolution, of an acyl-enzyme intermediate for imipenem bound to the TEM-1 $\beta$-lactamase from Escherichia coli, the parent enzyme of 67 clinical variants. The structure indicates an unprecedented conformational change for the complex which accounts for the ability of this antibiotic to resist hydrolytic deactivation by $\beta$-lactamases. Computational molecular dynamics underscored the importance of the motion of the acyl-enzyme intermediate, which may be a general feature for catalysis by these enzymes.
\end{abstract}

Assuming that the current efforts in the pharmaceutical companies toward discovery and development of novel antibiotics are successful, the use of these new agents would inevitably select for resistance in microbial populations in a relatively short time. ${ }^{1-3}$ It is critical that clinical selection of resistance be delayed as long as possible and that the principles for such resistance mechanisms be elucidated to help prolong the utility of existing drugs. ${ }^{4}$ One class of $\beta$-lactam antibiotics which has largely been resistant to the deleterious effects of the bacterial resistance enzymes is carbapenems,${ }^{5-8}$ a member of which, imipenem (1), has found clinical utility. ${ }^{9}$ Imipenem is often considered an antibiotic of last resort against the vancomycinresistant Gram-positive bacteria, as well as against multiple antibiotic-resistant Gram-negative organisms. The structural reason for this resistance of carbapenems to the action of $\beta$-lactamases is unknown, and it represents a subject which has yet to diminish in its biomedical importance.

Despite the high affinity of the enzyme for the drug, the poor turnover of imipenem (1) by class A $\beta$-lactamases stems only from a slow deacylation rate. Furthermore, the kinetics of

\footnotetext{
$\dagger$ Institut de Pharmacologie et de Biologie Structurale du CNRS.

$\doteqdot$ Wayne State University.

(1) Swartz, M. N. Proc. Natl. Acad. Sci. U.S.A. 1994, 91, 2420.

(2) Jones, R. N. Am. J. Med. 1996, 100 (suppl. 6A), 3S.

(3) Davies, J. E. Antibiotic resistance: origins, evolution, selection and spread; Ciba Foundation Symposium 207; Chadwick, D. J., Goode, J., Eds.; John-Wiley: Chichester, 1997; pp 15-35.

(4) Neu, H. C. Science 1992, 257, 1064

(5) Bush, K.; Jacoby, G. J. Antimicrob. Chemother. 1997, 39, 1.

(6) Kropp, H.; Gerckens, L.; Sundelof, J. G.; Kahan, F. M. Rev. Infect. Dis. 1985, 7 (suppl. 3), S389.

(7) Rasmussen, B. A.; Bush, K. Antimicrob. Agents Chemother. 1997, 41, 223.

(8) Matagne, A.; Lamotte-Brasseur, J.; Frère, J.-M. Biochem. J. 1998 330,581 .
}

(9) Balfour, J. A.; Bryson, H. M.; Brogden, R. N. Drugs 1996, 51, 99.

\section{Scheme 1}

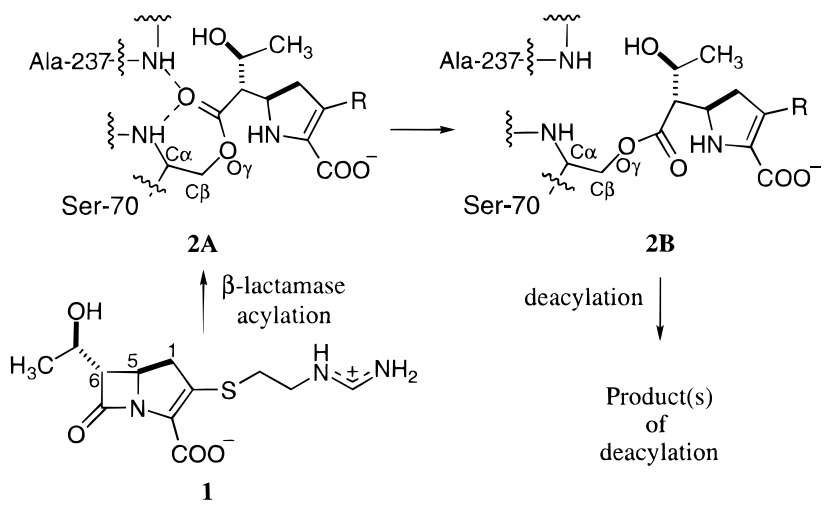

turnover are biphasic, suggestive of two distinct acyl-enzyme species. ${ }^{10}$ We report herein the crystal structure for one acylenzyme intermediate for imipenem with the TEM-1 $\beta$-lactamase, a common class A $\beta$-lactamase from Escherichia coli. Imipenem is found covalently bound to Ser-70 $\mathrm{O} \gamma$ (Figure 1A) as the $\Delta^{2}$ tautomer (Scheme 1).

The backbone atoms of the protein in the native enzyme and in the TEM-1-imipenem complex are identical within experimental errors (rmsd of $0.24 \AA$ ), except for residues 129-131,

(10) (a) Taibi, P.; Mobashery, S. J. Am. Chem. Soc. 1995, 117, 7600. (b) The value for $k_{\text {cat }}$ for the fast phase of the turnover of imipenem by the TEM-1 $\beta$-lactamase has been reported at $0.04 \mathrm{~s}^{-1}$ (ref 10a). Considering that the $k_{\text {cat }}$ values for the favorable substrates, such as certain penicillins, approach $2000 \mathrm{~s}^{-1}$, this difference amounts to 50000 -fold. The effect of this rate attenuation has been determined to be due to the $6 \alpha-1 R$ hydroxyethyl group of imipenem. Consistent with these determinations, studies with judiciously designed penicillanate derivatives have documented that the $6 \alpha-1 R$-hydroxyethyl group can lower the value of $k_{\text {cat }} / K_{\mathrm{m}}$ for the substrate of the TEM-1 $\beta$-lactamase by as much as 10000 -fold (Miyashita, K.; Massora, I.; Mobashery, S. Bioorg. Med. Chem. Lett. 1996, 6, 319). 

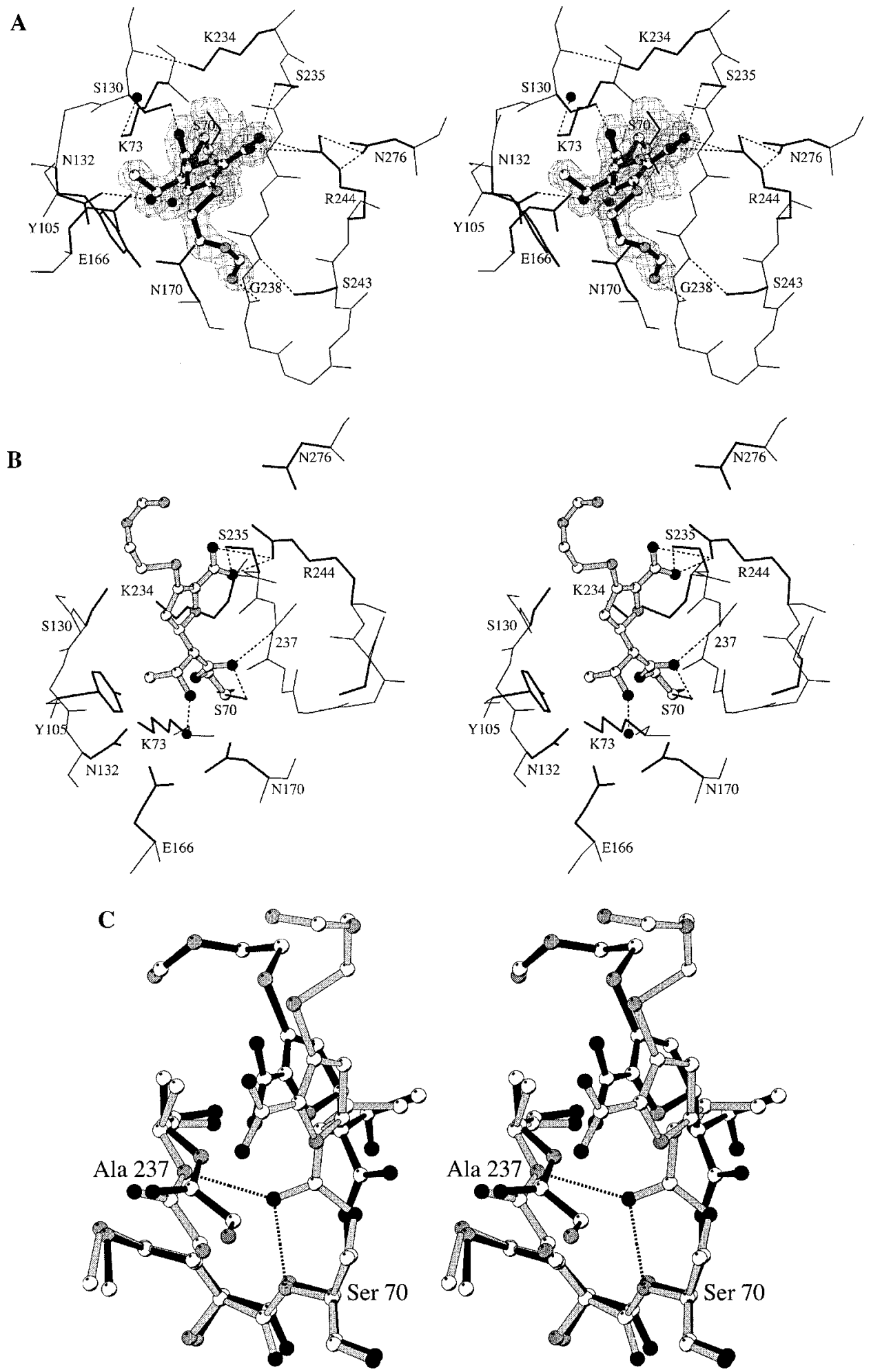

Figure 1. (A) Stereoview of the X-ray structure for the acyl-enzyme intermediate of imipenem with the TEM-1 $\beta$-lactamase shown in the final electron density map. Hydrogen bonds are represented as dotted lines. Water molecules are depicted as black spheres. (B) The energy-minimized structure for the canonical acyl-enzyme intermediate of imipenem with the TEM-1 $\beta$-lactamase. (C) The structures in (A) and (B) are superimposed here to demonstrate the motion of the acyl-enzyme species. The ester carbonyl of the canonical acyl-enzyme species (in gray) is held in the oxyanion hole by two hydrogen bonds (dotted lines) to the main-chain amines of Ser-70 and Ala-237. The acyl-enzyme species seen in the crystal structure is depicted in black.

237-244, and the $\mathrm{N}$ - and $\mathrm{C}$-terminal helices. The rms deviation on these main-chain regions are $1.5 \AA, 0.5,0.4$, and $0.5 \AA$, respectively. The conformation adopted by the $129-131$ region seems necessary to accommodate the $6 \alpha-1 R$-hydroxyethyl substituent of imipenem in the acyl-enzyme intermediate. The peptide bond between residues $129-130$ rotates by nearly $180^{\circ}$, and the carbonyl oxygen atom of residue 129 is hydrogen bonded to the $\mathrm{N} \xi$ atom of Lys-234 (3.1 $\AA$ ), which shifted by $1.4 \AA$ compared to the native enzyme (Figure 1A). Lys-234 is believed to provide a strong electrostatic pull in anchoring the carboxylate of the substrate(s). ${ }^{11}$ This role is substantially diminished in importance for the interaction of the enzyme with 

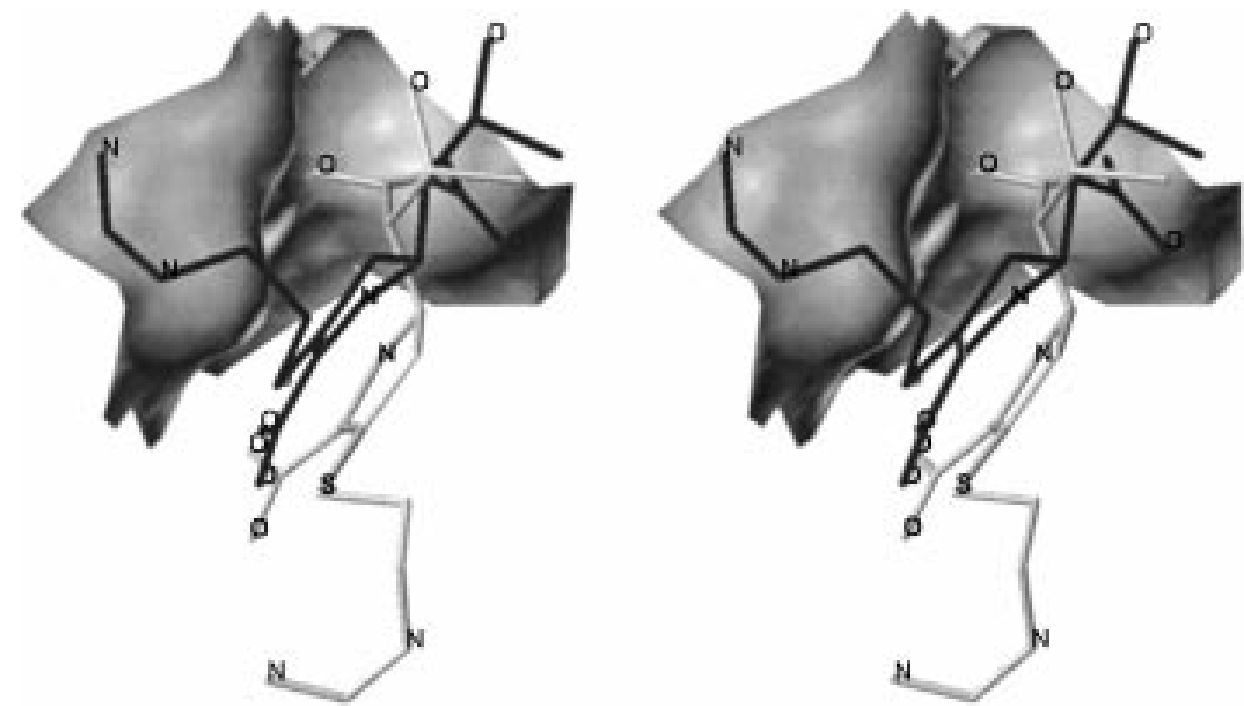

Figure 2. A different perspective for the two superimposed acyl-enzyme intermediates. A portion of the active site which encompasses the oxyanion hole is shown as a surface. The ester carbonyl of the canonical acyl-enzyme species (in gray) is shown housed in the oxyanion hole, and that for the species from the crystal structure is shown pointing out of it (in dark gray). The heteroatoms in imipenem are labeled.

imipenem, since the distance between the $\mathrm{N} \xi$ and the closest carboxylate oxygen of imipenem is $6.4 \AA$. The displacement of the loop 129-131 generated a cavity filled by a water molecule, which provides the single polar interaction to the essential Lys-73 (Figure 1A).

The main-chain atoms of residues 237-244 shifted toward the substrate binding cavity. A salt bridge interaction was observed between the side chain of Arg-244 and the carboxylate of imipenem (2.61 $\AA$ ). In this interaction, the carboxylate displaced the Arg-244 side chain away from the active site, a movement which propagated to helices H1 (residues 26-40) and H11 (residues 272-288), through the hydrogen bond between Arg-244 and Asn-276 (Figure 1A).

The conformation of the acyl-enzyme species of imipenem is unprecedented because the ester carbonyl oxygen was not located in the oxyanion hole (Figure 1A). It points toward Ser$130 \mathrm{O} \gamma$, at a distance of $3.3 \AA$. The hydroxyl group of the $6 \alpha-1 R$-hydroxyethyl substituent formed a hydrogen bond to Asn$132 \mathrm{O} \delta 1(2.7 \AA)$. The methyl group of the $6 \alpha-1 R$-hydroxyethyl substituent was found at van der Waals distance to the $\mathrm{C} \beta$ atom of Ser-130 and to the $C \delta 1$ atom of Tyr-105. This side chain is pointing toward the carbapenem and has rotated by 110 degrees away from its position seen in the several structures of this enzyme which have been determined. ${ }^{12-16}$

The hydrolytic water is present in the TEM-1-imipenem complex, and was merely displaced by $0.3 \AA$ from its position in the native enzyme. It formed a hydrogen bond to the hydroxyl group of the $6 \alpha-1 R$-hydroxyethyl substituent $(2.5 \AA)$, an interaction which likely would decrease its nucleophilicity.

In class A $\beta$-lactamases, activation of the carbonyl oxygen atom of the $\beta$-lactam ring for acylation of Ser-70 O $\gamma$ involves displacement of a water molecule from the oxyanion hole. This

(11) Ellerby, L. M.; Escobar, W. A.; Fink, A. L.; Mitchinson, C.; Wells, J. A. Biochemistry 1990, 29, 5797.

(12) Jelsch, C.; Mourey, L.; Masson, J. M.; Samama. J. P. Proteins Struct. Funct. 1993, 16, 364.

(13) Strynadka, N. C. J.; Adachi, H.; Jensen, S. E.; Johns, K.; Sielecki, A.; Betzel, C.; Sutoh, K.; James, M. N. Nature 1992, 359, 700.

(14) Fonzé, E.; Charlier, P.; Toth, Y.; Vermeire, M.; Raquet, X.; Dubus, A.; Frère, J.-M. Acta Crystallogr., Sect. D 1995, 51, 682.

(15) Strynadka, N. C. J.; Martin, R.; Jensen, S. E.; Gold, M.; Jones, J. B. Nature Struct. Biol. 1996, 3, 688.

(16) Maveyraud, L.; Massova, I.; Birck, C.; Miyashita, K.; Samama, J. P.; Mobashery, S. J. Am. Chem. Soc. 1996, 118, 7435. water molecule is not found in the oxyanion hole even though the ester carbonyl oxygen is not present in that position. The absence of water molecule in the oxyanion hole of the TEM1-imipenem acyl-enzyme complex implies that this water was excluded by imipenem in the acylation step, before the conformational rearrangement leading to the observed complex.

We generated a computational model for the canonical acylenzyme intermediate, the immediate product of Ser-70 acylation (Figure 1B), in which the ester carbonyl oxygen is positioned in the oxyanion hole (species $2 \mathrm{~A}$ in Scheme 1). We suggest that this immediate product of enzyme acylation is the species that undergoes the relatively more rapid initial hydrolysis, before transition to the second acyl-enzyme intermediate observed in the X-ray structure (species 2B in Scheme 1; Figure 1A). The $6 \alpha-1 R$-hydroxyethyl moiety in this model presents a steric and electrostatic barrier to the approach of the hydrolytic water to the ester carbonyl and would explain, consistent with the kinetic findings, that even in the more rapid phase of imipenem turnover, the rate of deacylation is slow compared to that for good substrates. ${ }^{10}$ The second acyl-enzyme intermediate, that revealed by the $\mathrm{X}$-ray structure, undergoes deacylation even more slowly than the first species. This species has to revert back to the canonical acyl-enzyme intermediate prior to deacylation. Figure 1C depicts the superimposition of the immediate acyl-enzyme intermediate (Figure 1B) and that seen in the X-ray crystal structure (Figure 1A).

Molecular dynamics simulations were carried out to gain an understanding of the rearrangement process. These simulations were performed on two acyl-enzyme species, (1) the computational structure for the canonical acyl-enzyme species of imipenem (Figure 1B) and (2) the same complex without the $6 \alpha-1 R$-hydroxyethyl group. In the complex of the enzyme with the variant of imipenem, the distances between the ester carbonyl oxygen and the backbone nitrogens of Ser-70 and Ala-237, which constitute the "oxyanion hole" (Figure 2), remain within good hydrogen-bonding range $(<3 \AA)$ for the entire period of simulation (Figure 3A). On the other hand, in the canonical TEM-imipenem complex the distance between the ester carbonyl and the Ser-70 amine increased to approximately $4 \AA$ between 37 and 38 ps of simulation, indicating that the hydrogen bond was lost (Figure 3B). The other hydrogen bond to the amine of Ala-237 also increased in length, going beyond $3 \AA$ 
Table 1. Summary of Crystallographic Analysis

Data Collection

\begin{tabular}{|c|c|c|c|c|c|}
\hline \multirow[b]{2}{*}{ resolution $(\AA)$} & \multicolumn{2}{|c|}{ reflections } & \multirow[b]{2}{*}{ completeness $^{a}$} & \multirow[b]{2}{*}{$\mathrm{I} /<\sigma \mathrm{I}>$} & \multirow[b]{2}{*}{$R_{\mathrm{sym}} b$} \\
\hline & measured & unique & & & \\
\hline $18-1.8$ & 84,646 & 21,770 & $98.3(96.8)$ & $20.9(8.7)$ & $5.9(11.6)$ \\
\hline
\end{tabular}

\begin{tabular}{|c|c|c|c|c|c|c|c|c|c|c|}
\hline \multicolumn{3}{|c|}{ atoms } & \multirow[b]{2}{*}{$R / R_{\text {free }}^{c}$} & \multicolumn{3}{|c|}{ temperature factors $\left(\AA^{2}\right)$} & \multicolumn{4}{|c|}{ rms deviations } \\
\hline protein & imipenem & solvent and ions & & protein & inhibitor & solvent & bonds $(\AA)$ & angles (deg) & dihedrals (deg) & impropers (deg) \\
\hline 2090 & 20 & 387 & $0.173 / 0.238$ & 9.50 & 13.47 & 21.42 & 0.010 & 1.9 & 23.4 & 1.6 \\
\hline
\end{tabular}

${ }^{a}$ Numbers in parentheses refer to the highest resolution shell $(1.89-1.80 \AA) .{ }^{b} R_{\mathrm{sym}}=\sum \sum\left|\langle I\rangle-I_{i}\right| / \sum \sum I_{i} \cdot{ }^{c} R=\sum|| F_{\mathrm{o}}|-| F_{c}|| / \sum\left|F_{\mathrm{o}}\right|$. The $R_{\mathrm{free}}$ value was calculated from a random set of reflections (10\%) which were omitted from structure refinement. The $\mathrm{R}$ value was calculated from the remaining reflections.
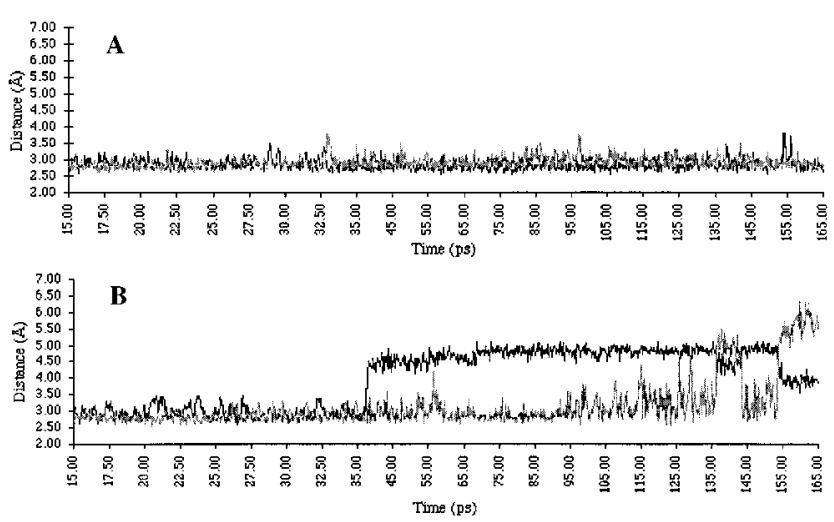

Figure 3. The distance versus time profiles for the dynamics simulations on (A) the immediate acyl-enzyme intermediates for the TEM-imipenem complex and (B) the TEM-imipenem analogue without the $6 \alpha-1 R$-hydroxyethyl side chain. Black and gray traces represent the profile of the distances between the main-chain amine of Ser-70 and the acyl oxygen and that of Ala- 237 and the acyl oxygen, respectively.

several times during the simulation after the initial 38 ps. After the initial $125 \mathrm{ps}$, these movements became more pronounced, and the ester moiety moved more freely for the remainder of the simulation. The simulation illustrated that the acyl-enzyme intermediate was able to move in and out of the oxyanion hole and, in essence, partition between the two intermediary acylenzyme species, as it should, on the basis of the known kinetic behavior for turnover of imipenem. ${ }^{10}$

We suggest that the torsional strain caused by the $6 \alpha-1 R$ hydroxyethyl substituent accounts for this molecular motion. The torsion angle of $\mathrm{C} \alpha-\mathrm{C} \beta-\mathrm{O} \gamma-\mathrm{C}$ is $95.5^{\circ}$ in the model of the canonical acyl-enzyme intermediate for imipenem (species $2 \mathrm{~A}$ in Scheme 1). In the $\mathrm{X}$-ray structure, this torsion angle is $-173.8^{\circ}$, which is effectively unstrained (species $2 \mathrm{~B}$ in Scheme 1). The driving force for this rearrangement is the presence of the methyl group of the $6 \alpha-1 R$-hydroxyethyl. Indeed, formation of the canonical acyl-enzyme intermediate for $6 \alpha$-hydroxymethylpenicillanate with TEM-1 induced no conformational change of the protein under the reported experimental conditions, and this crystal structure ${ }^{16}$ indicates that the decoration with the additional methyl group in the $6 \alpha-1 R$-hydroxyethyl substituent would induce steric encumbrance with protein atoms. This methyl group forces the structural adaptation observed in the TEM-1-imipenem complex and facilitates the transition to the second acyl-enzyme intermediate. This process is favored by the relative stability (slow rate of hydrolysis) of the canonical acyl-enzyme species, which likely provides the opportunity for this species to explore a different low-energy state.

The motion in and out of the oxyanion hole is quite intriguing and occurs in the simulations at the picosecond time scale, while the deacylation chemistry even for the more favorable substrates occurs at the millisecond time scale. ${ }^{17}$ It argues for the fact that the motion may be of a general nature, pertinent for discussion of the catalytic process, when considering the turnover of good and poor $\beta$-lactamase substrates, particularly in the deacylation step.

The flexibility in the structure of the active site that we have documented for the first time here with this crystal structure, in conjunction with the findings of the simulations, presents a dynamic picture for the turnover chemistry of $\beta$-lactams by $\beta$-lactamases, which heretofore was not appreciated in view of the structural aspects of the function of these enzymes.

\section{Experimental Section}

Methods. Crystals ${ }^{18}$ were soaked, at $4{ }^{\circ} \mathrm{C}$, in a saturated imipenem solution in $20 \mathrm{mM} \mathrm{Na}, \mathrm{K}$-phosphate, $\mathrm{pH} 7.8$, containing $50 \%$ of saturated ammonium sulfate. After $20 \mathrm{~min}$, half of the solution was replaced by a fresh saturated imipenem solution, and the soaking was pursued for another $20 \mathrm{~min}$. The crystal was mounted in a cryoloop, transferred in a 50\% (v/v) PEG 20 $\mathrm{K}$ solution for a few seconds and frozen in a $100 \mathrm{~K}$ nitrogen flux. Diffraction data were collected on a MarResearch imaging plate at the $\times 31$ synchrotron beamline of the EMBL outstation at DESY (Hamburg, Germany), with detector distance $120 \mathrm{~mm}$, wavelength $1.1271 \AA$ and frame size $1^{\circ}$. Data were processed using MOSFLM $5.40^{19}$ and CCP4. ${ }^{20}$ A rigid body refinement, using the TEM-1 structure (PDB entry $1 \mathrm{btl}$ ), was performed to account for the cell parameters variation between the native and derivatized crystals. All reflections between 18 and $1.8 \AA$ were used in refinement with REFMAC. ${ }^{21}$ An external bulk solvent contribution was computed with X-PLOR ${ }^{22}$ and updated after each major refinement cycle. The electron density map after the first refinement cycle allowed identification of all the substrate atoms. Water molecules were automatically introduced/ removed and refined using a procedure that alternates the use of REFMAC ${ }^{21}$ and ARP. ${ }^{23}$ The final crystallographic $R$ and

(17) Christensen, H.; Martin, M. T.; Waley, S. G. Biochem. J. 1990, $266,853$.

(18) Jelsch, C.; Lenfant, F.; Masson, J.-M.; Samama, J. P. J. Mol. Biol. 1992, 223, 377.

(19) Leslie, A. G. W. In Proceedings of the CCP4 Study Weekend; J. R. Heliwell, P. A. Machin, M. Z. Papiz, Eds.; Daresbury, Warrington WA4 4AD, UK, Daresbury Laboratory, 1987; pp 39-50.

(20) Collaborative Computational Project, Number 4. Acta Crystallogr. 1994, D50, 760 .

(21) Murshudov, G. N.; Vagin, A. A.; Dodson, E. J. Acta Crystallogr. 1997, D53, 240 .

(22) Brünger, A. T. X-PLOR version 3.1: A system for X-ray crystallography and NMR; Yale University Press: New Haven, CT, 1992.

(23) Lamzin, V. S.; Wilson, K. S. Acta Crystallogr. 1993, D49, 129. 
$R_{\text {free }}{ }^{24}$ values are 0.173 and 0.238 , respectively. The final model comprises all proteins and substrate atoms, 8 sulfate ions, and 347 water molecules. Alternate conformations were assigned to 13 side chains (Table 1 ).

The energy minimization procedures and molecular dynamics simulations were performed using the AMBER 4.1 package. $^{25}$ In all starting conformations, the ester oxygen in the canonical acyl-enzyme species was in the oxyanion hole, making hydrogen bonds to the backbone nitrogens of Ser-70 and Ala237. The crystallographic water molecules were retained and the complexes were immersed in a shell of water of $12 \AA$ thickness from the surface of the enzyme complex. The energyminimized (10 000 cycles) complexes were used as the starting

(24) Brünger, A. T. Nature 1992, 355, 472.

(25) Weiner, S. J.; Kollman, P. A.; Case, D. A.; Singh, U. C.; Ghio, C.; Alagona, G.; Profeta, S., Jr.; Weiner, P. A. J. Am. Chem. Soc. 1984, 106, 765 . conformations for the molecular dynamics simulations. The structures were warmed from 0 to $300 \mathrm{~K}$ in steps of $20 \mathrm{~K}$ per 1 ps. Simulations were carried out for 165 ps for each of the two complexes at $300 \mathrm{~K}$. The coordinates were collected for every $0.05 \mathrm{ps}$ during the first $20 \mathrm{ps}$, and for every $0.2 \mathrm{ps}$ thereafter. They were measured using the CARNAL module in AMBER package and plotted as a function of time.

Acknowledgment. We thank Stéphanie Cabantous for excellent technical assistance. The work in France was funded in part by INSERM (CRE contract 930612; J.P.S.) and the Regional Midi-Pyrenees (contract 9200843; J.P.S.). The work in the U.S.A. was supported by grants from the National Institutes of Health and the National Science Foundation (S.M.). The crystallographic coordinates are deposited in the Brookhaven Protein Data Bank.

JA9818001 